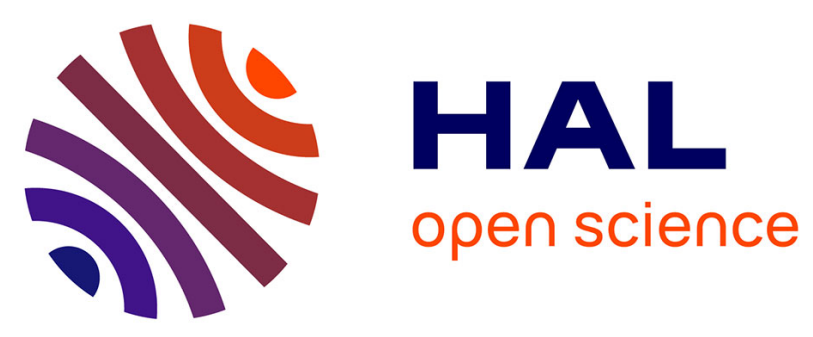

\title{
The Imaging Modulography Technique Revisited for High-Definition Intravascular Ultrasound: Theoretical Framework
}

\author{
Antoine Tacheau, Simon Le Floc'H, Gérard Finet, Marvin M Doyley, Roderic \\ Pettigrew, Guy Cloutier, Jacques Ohayon
}

\section{To cite this version:}

Antoine Tacheau, Simon Le Floc'H, Gérard Finet, Marvin M Doyley, Roderic Pettigrew, et al.. The Imaging Modulography Technique Revisited for High-Definition Intravascular Ultrasound: Theoretical Framework. Ultrasound in Medicine \& Biology, 2016, 42 (3), pp.727-741. 10.1016/j.ultrasmedbio.2015.11.015 . hal-01465820

\author{
HAL Id: hal-01465820 \\ https://hal.science/hal-01465820
}

Submitted on 13 Feb 2017

HAL is a multi-disciplinary open access archive for the deposit and dissemination of scientific research documents, whether they are published or not. The documents may come from teaching and research institutions in France or abroad, or from public or private research centers.
L'archive ouverte pluridisciplinaire $\mathbf{H A L}$, est destinée au dépôt et à la diffusion de documents scientifiques de niveau recherche, publiés ou non, émanant des établissements d'enseignement et de recherche français ou étrangers, des laboratoires publics ou privés. 


\title{
THE IMAGING MODULOGRAPHY TECHNIQUE REVISITED FOR HIGH- DEFINITION INTRAVASCULAR ULTRASOUND: THEORETICAL FRAMEWORK
}

\author{
Antoine Tacheau, $*$ Simon Le Floc’h, ${ }^{\dagger}$ Gérard Finet, ${ }^{\ddagger}$ Marvin M. Doyley, \\ Roderic I. Pettigrew, ${ }^{\Uparrow}$ Guy Cloutier, and Jacques Ohayon *\# \\ *Laboratory TIMC-IMAG/DyCTiM, UJF, CNRS UMR 5525, Grenoble, France; ${ }^{\dagger}$ Laboratory LMGC, CNRS UMR 5508 , \\ University of Montpellier II, Montpellier, France; ${ }^{\ddagger}$ Department of Hemodynamics and Interventional Cardiology, Hospices \\ Civils de Lyon and Claude Bernard University Lyon, INSERM Unit 886, Lyon, France; ${ }^{\S}$ Department of Electrical and Computer \\ Engineering, University of Rochester, Rochester, New York, USA; ${ }^{\circledR}$ Laboratory of Integrative Cardiovascular Imaging Science, \\ National Institute of Diabetes, Digestive, and Kidney Diseases, National Institutes of Health, Bethesda, Maryland, USA; \\ " Laboratory of Biorheology and Medical Ultrasonics, University of Montreal Hospital Research Center (CRCHUM), Montréal, \\ Québec, Canada; and ${ }^{\#}$ University Grenoble-Alpes, Polytech Annecy-Chambéry, Le Bourget du Lac, France
}

\begin{abstract}
Mechanical characterization of atherosclerotic lesions remains an essential step for the detection of vulnerable plaques (VPs). Recently, an intravascular ultrasound (IVUS) elasticity reconstruction method (iMOD) has been tested in vivo by our group. The major limitation of iMOD is the need to estimate the strain field in the entire VP despite attenuated depth penetration signals when using high-definition (HD) IVUS systems. Therefore, an extended iMOD approach (E-iMOD) was designed and applied to coronary lesions of patients imaged in vivo with IVUS. The E-iMOD method (i) quantified necrotic core areas with a mean absolute relative error of $3.5 \pm 3.5 \%$ and (ii) identified Young's moduli of the necrotic cores and fibrous regions with mean values of $5.7 \pm 0.8 \mathrm{kPa}$ and $794.5 \pm 22.0 \mathrm{kPa}$ instead of $5 \mathrm{kPa}$ and $800 \mathrm{kPa}$, respectively. This study demonstrates the potential of the improved HD-IVUS modulography technique E-iMOD to characterize coronary VPs. (E-mail: jacques.ohayon@imag.fr)
\end{abstract}

Key Words: Elastography, Modulography, Linear elasticity, Inverse problem, Vulnerable plaques, Coronary disease.

\section{INTRODUCTION}

Atherosclerotic coronary plaque rupture and subsequent thrombosis is the leading cause of acute coronary syndrome and responsible for the majority of cardiovascular deaths (Fleg et al. 2012; Go et al. 2013; Lloyd-Jones et al. 2010). Vulnerable plaques (VPs; plaques likely to rupture) possess specific geometrical (Virmani et al. 2006), mechanical (Cheng et al. 1993; Loree et al. 1992; Ohayon et al. 2001; Riou et al. 2014) and biological (Broisat et al. 2011) features. An early and accurate determination of these properties remains an essential step to implementing preventive therapeutic strategies (Libby 2001).

Address correspondence to: Professor Jacques Ohayon, Laboratory TIMC-IMAG/DyCTiM, UJF, CNRS UMR 5525, Grenoble, France. E-mail: jacques.ohayon@imag.fr
Studies have shown that fibrous cap thickness index $(<65 \mu \mathrm{m})$ alone is not a sufficient predictor of plaque rupture (Cardoso et al. 2014; Ohayon et al. 2008; Virmani et al. 2000). Biomechanical studies have identified peak cap stress (PCS) amplitude as an additional key predictor of plaque disruption (Finet et al. 2004; Ohayon et al. 2014). In vivo imaging modalities are needed to characterize and identify specific biomechanical factors responsible for plaque instability and rupture (Fleg et al. 2012; Magnoni et al. 2015). Following the spirit of Ophir and colleagues (Cespedes et al. 1993; Ophir et al. 1991), several intravascular ultrasound (IVUS) strain-elastography (de Korte et al. 2002; Maurice et al. 2004; Richards and Doyley 2013) and strain-palpography (Céspedes et al. 2000; Deleaval et al. 2013; Schaar et al. 2005) reconstruction techniques were developed to characterize atherosclerotic coronary lesions and to predict their vulnerability to rupture. IVUS 
strain-elastography approaches were developed to highlight the spatial strain distribution (i.e., strainelastogram) over the entire vessel wall (KeshavarzMotamed et al. 2014; Majdouline et al. 2014; Maurice et al. 2007; Richards and Doyley 2013) or over a restricted thick endoluminal region (de Korte et al. 2002; Doyley et al. 2001). Such IVUS imaging techniques based on the optical flow (KeshavarzMotamed et al. 2014; Majdouline et al. 2014; Maurice et al. 2004), time-delay correlation estimation (de Korte et al. 2002) or a non-rigid image-registration method (Richards and Doyley 2013) allowed the calculations of intraplaque strain images during the cardiac cycle. However, these methods did not overcome the main limitation related to the complex morphologies of atherosclerotic lesions, which alter the intraplaque strain fields and inhibit direct translation into intra-parietal stress and plaque mechanical properties.

Young's modulus reconstruction of atherosclerotic plaque (i.e., modulogram), based on the strain field measurements, remains a challenge that has been pursued by a variety of methods (Baldewsing et al. 2008; Doyley 2012; Le Floc'h et al. 2009). Following the spirit of Baldewsing et al. (2005) and Doyley's works (Doyley 2012; Richards and Doyley 2011), our group (Bouvier et al. 2013; Le Floc'h et al. 2009, 2010, 2012) demonstrated that by pre-conditioning the algorithm based on the best estimation of plaque component boundaries, we could improve the elasticity reconstruction. An original pre-conditioning step to extract the plaque morphology and an iterative approach combining a dynamic watershed segmentation method with an optimization procedure were proposed to highlight modulograms of human coronary atherosclerotic lesions (this approach was named iMOD for imaging modulography; Le Floc'h et al. 2012). However, one major limitation of such a method is the need to estimate accurately the strain field in the entire lesion. This may be difficult as the amplitude of the original signal becomes attenuated as the depth of penetration increases and shadow artifacts produced by calcified nodules impair imaging of deep plaque structures. Strain-elastograms are also difficult to reconstruct when using intravascular imaging techniques with limited depth penetration signals, such as optical coherence tomography (OCT) or the new generation of highdefinition intravascular ultrasound (HD-IVUS) imaging systems equipped with high-frequency catheters $\geq 60$ MHz (Kobayashi et al. 2014; Waters et al. 2011).

Therefore, the present biomechanical study was designed to extend the theoretical framework of the iMOD technique when considering HD-IVUS catheters with limited depth penetration. This extended iMOD technique (E-iMOD), based on the continuum mechanics theory prescribing the strain field in the limited endoluminal region, was successfully applied to 10 VP morphologies: three modeled plaque geometries and seven coronary lesions of patients imaged in vivo with IVUS. The robustness and performance of the new elasticity reconstruction technique E-iMOD was investigated with regard to noise, which may affect prediction of plaque vulnerability.

\section{MATERIALS AND METHODS}

To test the numerical performance of the proposed E-iMOD algorithm, the finite element (FE) method was used to generate our input set of the intraplaque displacement and strain fields. As realistic human VP geometries were needed to perform the FE simulations, we used VP geometries of patients imaged in vivo with an IVUS system operating at $40 \mathrm{MHz}$. To simulate the acquisitions that we would obtain with limited depth penetration HD-IVUS catheters, the resulting FE displacement and strain fields computed in the endoluminal plaque regions and the luminal contours were the only inputs of our inverse model. Let us notice that the plaque component's contours were not given to solve the inverse problem.

\section{IVUS study and plaque geometries}

To study the performance of the proposed E-iMOD method, we used a patient population including five plaque geometries considered in a previous work conducted by our group on the elasticity-palpography technique (Deleaval et al. 2013). This will allow us also to discuss the complementarities of these two approaches when applied to the same VP morphologies.

Patient population. Arteries were explored in seven patients with stable angina and referred for percutaneous coronary intervention (PCI) at the Hospital of Cardiology and Pneumology of Lyon. Investigations were approved by institutional board of the hospital's cardiology department, and consent was obtained from the patients.

Intravascular ultrasound imaging. The dataset of non-ruptured VP geometries was obtained from systematic IVUS scans of the left main, left anterior descending and left circumflex coronary arteries following the protocol described by Rioufol et al. (2002). The recorded cross-sectional IVUS images corresponded to the sites exhibiting the thinner fibroatheroma cap. An iLab IVUS system (Boston Scientific, Watertown, MA, USA) equipped with 40-MHz catheters (Atlantis SR Pro 3.6F, Boston Scientific) was used for these clinical investigations.

IVUS image analysis. The IVUS echogenecity aspects were used to characterize atherosclerotic VP components. Anechogenic, homogeneous reflective and bright zones indicate presence of lipid or cellular deposition, organized or disorganized fibrosis and calcified regions, respectively (Di Mario et al. 1998). A manual 
segmentation procedure using ImageJ software (ImageJ, National Institutes of Health, Bethesda, MD, USA) was performed by a cardiologist to extract the contours of each plaque component. Coronary geometric configurations of atherosclerotic lesions acquired from this patient population were used to initiate our FE analysis.

IVUS measurements and definitions. Each crosssectional IVUS image of a lesion was quantitatively analyzed. Measurements were made for plaque area $\left(\mathrm{Pla}_{\text {area }}, \mathrm{mm}^{2}\right)$; lumen area $\left(\mathrm{Lu}_{\text {area }}, \mathrm{mm}^{2}\right)$; necrotic core area $\left(\mathrm{Core}_{\text {area }}, \mathrm{mm}^{2}\right)$; calcified area $\left(\mathrm{Cal}_{\text {area }}, \mathrm{mm}^{2}\right)$; degree of stenosis $\left(\right.$ Stenos $\left._{\mathrm{deg}}, \%\right)$, defined as $100 \times$ $\mathrm{Pla}_{\text {area }} /\left(\mathrm{Pla}_{\text {area }}+\mathrm{Lu}_{\text {area }}\right)$; and cap thickness $\left(\mathrm{Cap}_{\text {thick}}\right.$, $\mathrm{mm}$ ), defined as the shortest distance between the lumen and the necrotic core.

Idealized plaque geometries. In addition to the initial data set of VPs acquired on patients by IVUS, idealized eccentric VP geometries were drawn to obtain a series of VP geometries mimicking the plaque evolution. From one real plaque geometry acquired on a patient by IVUS (Fig. 1, plaque \#3), three idealized models mimicking the plaque evolution by increasing Cap thick and decreasing the necrotic core size were designed by a cardiologist (Fig. 1, plaques \#M1 to M3).

\section{Forward problem: Endoluminal displacement and radial strain fields}

The digitized contours obtained with ImageJ were imported into MATLAB (The MathWorks, Natick, MA, USA) and then transferred in the FE software COMSOL (Structural Mechanics Module, version 3.5, COMSOL Inc., Grenoble, France) via the LiveLink MATLAB/Comsol toolbox. The mechanical properties of the fibrous regions, calcified inclusions and soft necrotic cores were modelled as isotropic (Williamson et al. 2003) and quasi-incompressible media (Poisson's ratio $\nu=0.49$ ) with Young's moduli $\mathrm{E}_{\text {fibrosis }}=800 \mathrm{kPa}, \mathrm{E}_{\text {calcified }}=$ $5000 \mathrm{kPa}$ and $\mathrm{E}_{\text {core }}=5 \mathrm{kPa}$, respectively (Finet et al. 2004). Displacement and radial strain distributions were obtained by performing static FE computations on all VP geometries. The plane strain condition was assumed because only one 2-D IVUS image of the crosssectional section with a large stenosis was acquired in routine imaging on each patient during the clinical protocol. As instantaneous pressure was not recorded during the IVUS scans of the coronary arteries, a realistic pressure gradient $\Delta \mathrm{P}$ of $1 \mathrm{kPa}$ (or $7.5 \mathrm{~mm} \mathrm{Hg}$ ) occurring between two successive IVUS images was assumed (Le Floc'h et al. 2012). The entire plaque geometries were meshed with approximately 20,000 6-Node triangular elements. The center of mass of the lumen was used as the origin of the cylindrical coordinate system $(\mathrm{r}, \theta)$. The computed displacement and radial strain fields were interpolated on a regular polar mesh with a given radial step resolution of $15.4 \mu \mathrm{m}$ and an angular step resolution of 256 radial directions, which is the resolution one can expect from IVUS (Maurice et al. 2007). To simulate the acquisitions conducted with limited depth penetration IVUS catheters, the performance of the proposed elasticity reconstruction method E-iMOD was tested with displacement and strain fields computed in the endoluminal plaque regions $\Omega_{\text {endo }}$ only. For this study, we called them abusively "measured" displacement vector and strain fields ( $\vec{u}^{\text {meas }}$ and $\varepsilon_{r r}^{\text {meas }}$ ).

\section{Inverse problem: Governing equations and elasticity reconstruction method}

The endoluminal domain $\Omega_{\text {endo }}$ was assumed to be isotropic, heterogeneous and quasi-incompressible (Poisson's ratio $\nu=0.49$ ) and was described by the linear elastic Hooke's law:

$$
[\sigma]=\frac{E(\vec{x})}{(1+\nu)}\left(\frac{\nu}{(1-2 \nu)} \operatorname{Trace}[\varepsilon][I]+[\varepsilon]\right) \text { in } \Omega_{\text {endo }}
$$

where $[\sigma]$ and $[\varepsilon]$ are the stress and strain tensors, respectively; $[I]$ is the identity matrix; and E the Young's modulus, which is an arbitrary function of the position vector $\vec{x}$.

Assuming quasi-static condition and neglecting gravity and inertial forces, the stress tensor $[\sigma]$ satisfies the local static equilibrium equation:

$$
\nabla \cdot[\sigma]=\overrightarrow{0} \quad \text { in } \Omega_{\text {endo }}
$$

Assuming plane strain condition, the mathematical problem is correctly defined because the boundary conditions, at the inner $\left(\partial \Omega_{\text {endo }}^{\text {in }}\right)$ and outer $\left(\partial \Omega_{\text {endo }}^{\text {out }}\right)$ limits of the endoluminal domain $\Omega_{\text {endo }}$ (see Fig. 1, row 2), are given in terms of imposed stress and displacement. A blood pressure was imposed at the entire luminal limit $\left(\partial \Omega_{\text {endo }}^{\text {in }}\right)$ of the endoluminal domain of interest. At the free outer limit ( $\left.\partial \Omega_{\text {endo }}^{\text {out }-\overline{\text { Plaque }}}\right)$ of this domain of interest, a zero pressure was assumed, while at the intraplaque outer limit $\left(\partial \Omega_{\text {endo }}^{\text {out }- \text { Plaque }}\right)$ of the endoluminal domain, displacement was imposed. Such boundary conditions are realistic because (i) the intraplaque displacement field could be estimated by using the robust IVUSLagrangian speckle model estimator (Maurice et al. 2004) and (ii) the blood pressure could be recorded during the IVUS scans. A blood pressure of amplitude $\Delta \mathrm{P}$ (equal to $1 \mathrm{kPa}$ ) was applied on the inner boundary $\partial \Omega_{\text {endo }}^{\text {in }}$ :

$$
[\sigma] \vec{n}=-\Delta P \vec{n} \quad \text { on } \partial \Omega_{\text {endo }}^{\text {in }}
$$

where $\vec{n}$ is the external unit vector normal to the contour $\partial \Omega_{\text {endo }}^{\text {in }}$. Knowing the displacement field in the entire 

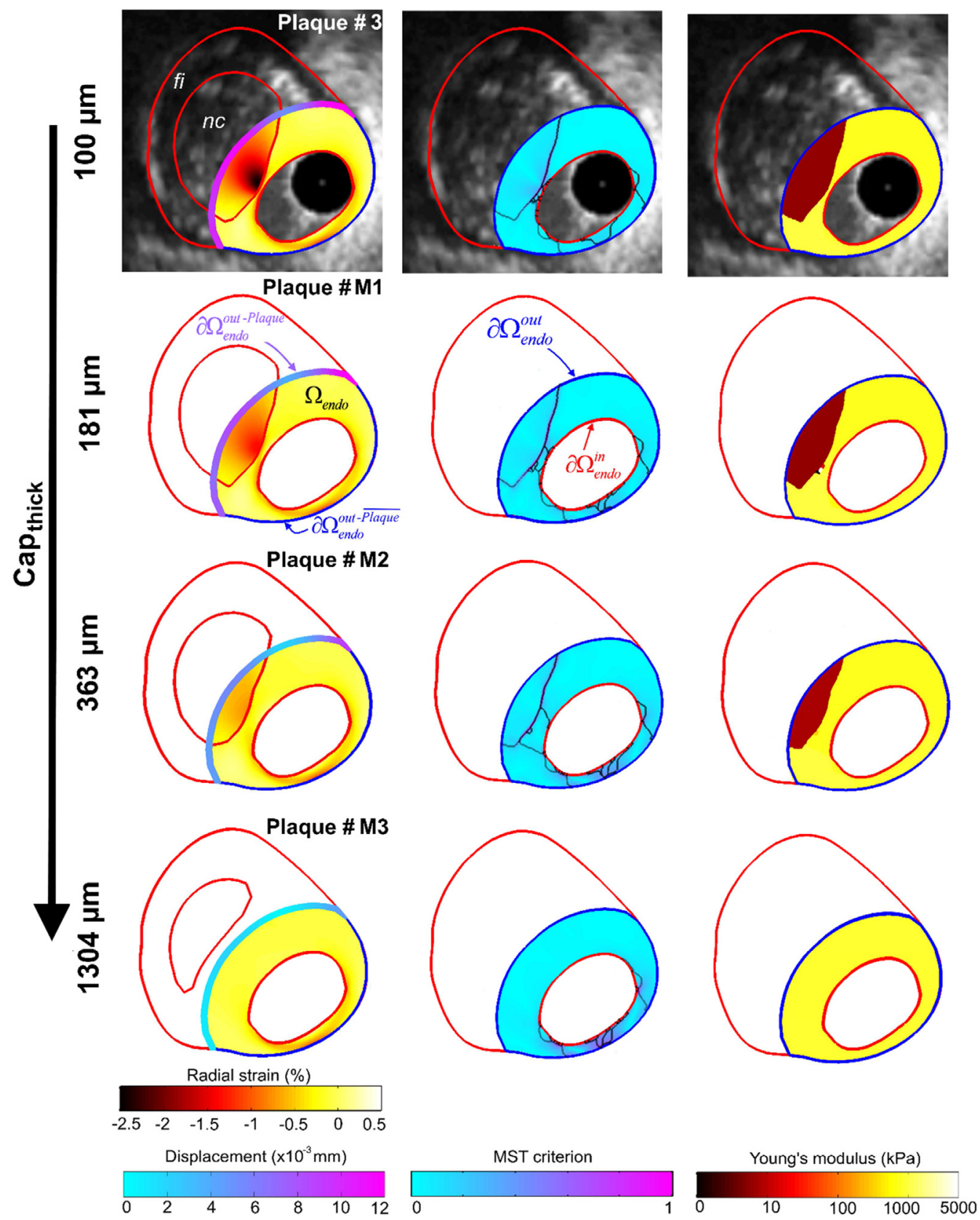

Fig. 1. Performance of the E-iMOD method to estimate cap thickness and to detect a vulnerable plaque when only a part of the large necrotic core is included in the endoluminal plaque domain $\Omega_{\text {endo }}$. Plaques \#3 and M1 to M3 were used for these simulations. Column 1: Input radial strain-elastogram and amplitude of the displacement vector applied on the intraplaque outer endoluminal boundary $\partial \Omega_{\text {endo }}^{\text {out }- \text { plaque }}$. Column 2: Spatial distribution of the normalized modified Sumi's transform (MST) criterion dW (see Eq. [5]) and the extracted inclusions contours $(\mathrm{n}=20)$ obtained from the combined MST-DWS procedure. Column 3: Resulting Young's modulus maps (i.e. modulograms) of the endoluminal region. Red lines delimit inclusions, lumen and external plaque contours. The blue contours delimit the endoluminal plaque domain $\Omega_{\text {endo }}$ of internal and external boundaries $\partial \Omega_{\text {endo }}^{\text {in }}$ and $\partial \Omega_{\text {endo }}^{\text {out }}$, respectively. nc $=$ necrotic core. fi $=$ fibrous region. The forward problem was conducted with fibrosis and necrotic core Young's moduli equal to $800 \mathrm{kPa}$ and $5 \mathrm{kPa}$, respectively.

endoluminal region $\Omega_{\text {endo }}$, we can express the following displacement-condition at the intraplaque outer endoluminal boundary $\partial \Omega_{\text {endo }}^{\text {out }-P \text { laque }}$ :

$$
\vec{u}=\vec{u}_{0}^{\text {meas }} \text { on } \partial \Omega_{\text {endo }}^{\text {out }-P \text { laque }}
$$

where $\vec{u}_{0}^{\text {meas }}$ is a known displacement vector field. The remaining complementary endoluminal boundary $\partial \Omega_{\text {endo }}^{\text {out } \overline{\text { Plaque }}}$ is assumed to be free of stress:

$$
[\sigma] \vec{n}=\overrightarrow{0} \quad \text { on } \partial \Omega_{\text {endo }}^{\text {out }-\overline{\text { Plaque }}}
$$


where $\partial \Omega_{\text {endo }}^{\text {out }}=\partial \Omega_{\text {endo }}^{\text {out }-P \text { laque }} \cup \partial \Omega_{\text {endo }}^{\text {out }-\overline{\text { Plaque }}}$.

The theoretical frameworks of the segmentation technique and the mathematical optimization procedure used have been described earlier (Le Floc'h et al. 2009). Briefly, considering two successive frames (at time $t_{j-1}$ and $t_{j}$ ) of the IVUS sequence, we applied the extended imaging modulography technique E-iMOD, which involves three successive steps: (i) the computation of a local pseudo-gradient elasticity amplitude $\mathrm{dW}$ computed on the defined regular polar mesh:

$$
d W=-\frac{1}{\varepsilon_{r r}}\left(\frac{\partial \varepsilon_{r r}}{\partial r}+\frac{2 \varepsilon_{r r}}{r}\right) d r-\frac{1}{\varepsilon_{r r}} \frac{\partial \varepsilon_{r r}}{\partial \theta} d \theta
$$

(this equation from here on is referred to as the "Modified Sumi's Transform" (MST) criterion); (ii) the dynamic watershed segmentation (DWS) procedure that makes use of the previous step's results to extract the inclusions' contours; and finally (iii) the mathematical optimization procedure that provides the estimated Young's moduli of detected inclusions and surrounding tissue. This last step relies on using the adjoint method to estimate, in the endoluminal domain $\Omega_{\text {endo }}$, the gradient of the normalized root mean squared error $\left(\mathrm{RMS}_{\text {error }}\right)$ between the measured $\varepsilon_{r r}^{\text {meas }}, u_{r}^{\text {meas }}, u_{\theta}^{\text {meas }}$ and computed $\varepsilon_{r r}^{E-i M O D}$, $u_{r}^{E-i M O D}, u_{\theta}^{E-i M O D}$ radial strains, and radial and circumferential displacements, respectively:

$$
R M S_{\text {error }}\left(t_{j}\right)=\sqrt{\sum_{k=1}^{3} \sum_{\text {elt }=1}^{N} \Delta \mathrm{S}_{\mathrm{elt}}^{2}\left(\chi_{k, \text { elt }}^{\text {mean }}-\chi_{k, \text { elt }}^{E-i M O D}\right)^{2} /\left(\chi_{k}^{\text {mean }}\right)^{2}}
$$

$$
\text { with } \chi_{k}^{\text {mean }}=\sum_{\text {elt }=1}^{N} \Delta \mathrm{S}_{\mathrm{elt}} \chi_{k, \text { elt }}^{\text {meas }}
$$

where $N$ is the total number of finite elements, $\Delta S_{\text {elt }}$ is the area of FE elt and $\chi_{k}=\varepsilon_{r r}, u_{r}, u_{\theta}$ for $\mathrm{k}=1,2,3$, respectively. The watershed (Watershed function, Imaging ToolBox, MATLAB, version 7.6, the MathWorks) and minimization procedures (Optimization Lab Module, COMSOL, version 3.5, COMSOL Inc.) were repeated with an increasing number $n$ of pre-conditioning regions to take into account smaller heterogeneity sizes. We assumed that a satisfactory solution was reached when the gradient-based optimization procedure reached a tolerance termination value lower than $10^{-6}$. During the optimization procedure, local Young's moduli were constrained to remain positive and between $1 \mathrm{kPa}$ and $10^{4} \mathrm{kPa}$. Sensitivity study with regards to noise on input radial
strain and displacement data

To investigate the influence of the noise on the performance of the new E-iMOD algorithm, white noise was added to each FE simulated radial strain and displacement fields used as input. A normal distribution of noise with zero mean and a standard deviation of $(a \Psi+b) \beta$, where $\Psi$ is either the radial displacement, circumferential displacement or radial strain, was used (Baldewsing et al. 2005; Le Floc'h et al. 2009). Values of factors $a$ and $b$ were fixed to $0.2 \%$ and $0.04 \%$, respectively, to obtain significant noise levels. The noise field was significantly amplified by increasing $\beta$ from 1 to 6 . For each level of noise, 20 computations in which the noise was spatially randomly distributed were performed and the reconstructed averaged Young's modulus constituents, necrotic core area and Cap thick amplitudes ( \pm standard deviations) of one VP were presented. We converted our white noise amplitude in $\mathrm{dB}$ scale based on the definition of the signal-to-noise ratio (see Appendix II of Deleaval et al. 2013).

Unless otherwise stated, all numerical results presented in our figures were derived (i) from measured radial strain $\varepsilon_{r r}^{\text {meas }}$ and displacement vector $\vec{u}^{\text {meas }}$ fields with no white noise (i.e., with $\beta=0$ ), (ii)

Table 1. Characteristics of the entire atherosclerotic plaques detected by intravascular ultrasound (IVUS) and modeled. To test

\begin{tabular}{|c|c|c|c|c|c|c|c|c|}
\hline & \multirow[b]{2}{*}{ Origin of geometry } & \multirow[b]{2}{*}{ Cap thickness $(\mu \mathrm{m})$} & \multicolumn{5}{|c|}{ Area $\left(\mathrm{mm}^{2}\right)$} & \multirow[b]{2}{*}{ Stenosis $(\%)$} \\
\hline & & & First necrotic core & Second necrotic core & Calcified & Plaque & Lumen & \\
\hline Plaque 2 & IVUS & 93 & 1.36 & - & - & 8.74 & 2.79 & 76 \\
\hline Plaque 3 & IVUS & 100 & 5.39 & - & - & 16.70 & 3.24 & 84 \\
\hline Plaque M1 & Model & 181 & 5.14 & - & - & 16.70 & 3.24 & 84 \\
\hline Plaque M2 & Model & 363 & 4.68 & - & - & 16.70 & 3.24 & 84 \\
\hline Plaque M3 & Model & 1304 & 2.57 & - & - & 16.70 & 3.24 & 84 \\
\hline Plaque 4 & IVUS & 162 & 0.51 & 1.20 & - & 17.85 & 6.75 & 73 \\
\hline Plaque 5 & IVUS & $<90(41)$ & 2.11 & - & 0.40 & 17.53 & 3.60 & 83 \\
\hline Plaque 6 & IVUS & $<90(62)$ & 3.54 & - & 1.60 & 15.64 & 4.52 & 78 \\
\hline Plaque 8 & IVUS & $<90(52)$ & 1.34 & 0.44 & - & 15.06 & 5.72 & 72 \\
\hline Plaque 9 & IVUS & $<90(50)$ & 0.87 & - & - & 8.74 & 3.74 & 70 \\
\hline
\end{tabular}
the E-iMOD's performance, cap thicknesses were randomly assigned (value in parentheses) when found to be under the limit of resolution obtained with the 40-MHz IVUS catheter (i.e., $<90 \mu \mathrm{m}$ ) 
with a blood pressure differential $\Delta \mathrm{P}$ of $1 \mathrm{kPa}$, (iii) by using the objective function defined previously in Eq. (6) with $\mathrm{k}=1,2,3$ and (iv) by performing the MST-DWS procedure to extract the inclusions' contours.

\section{RESULTS}

\section{IVUS study}

Table 1 provides the geometrical characteristics of the seven non-ruptured VPs scanned in vivo (plaques \#2,3 and 9 are three VPs without calcification but with one necrotic core; plaques \#4 and 8 are two VPs without calcification but with two necrotic cores; and plaques \#5 and 6 are two VPs with one necrotic core and one calcified inclusion) and the three modeled VPs (plaques \#M1 to M3) mimicking the lesion's growth.

\section{Influence of the displacement boundary condition on plaque elasticity reconstruction}

To highlight the importance of considering all displacement components to express the boundary condition at the intraplaque outer endoluminal frontier $\partial \Omega_{\text {endo }}^{\text {out }-P \text { laque }}$, we compared the modulograms computed when using all displacement components $u_{r}$ and $u_{\theta}$ with those computed when considering only $u_{r}$ To distinguish the error induced by the imposed boundary condition, the inverse problem simulations were conducted with real VP morphologies and with only $\varepsilon_{r r}^{\text {meas }}$. We found that the estimated Young's moduli of all necrotic cores and fibrous
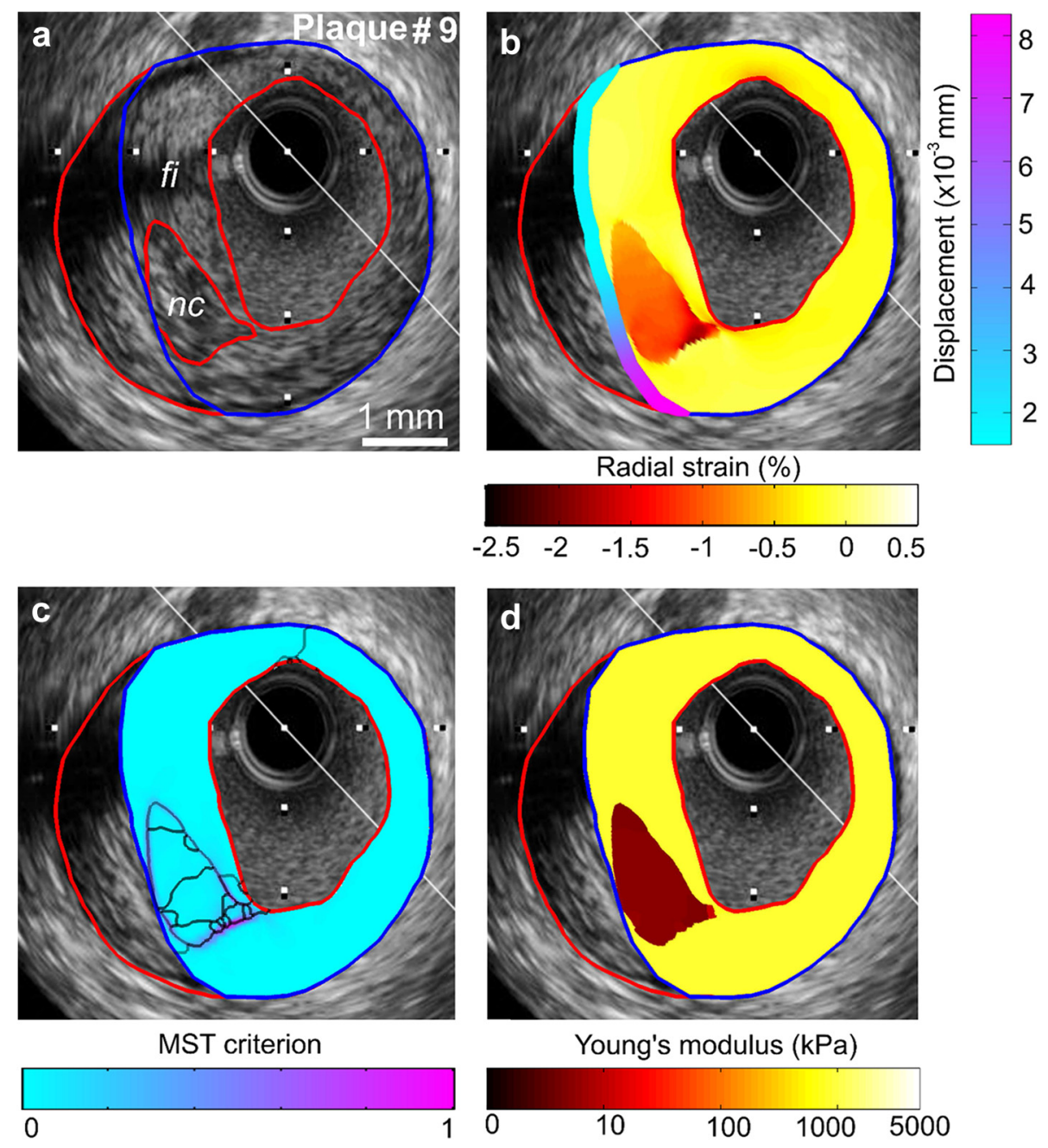

Fig. 2. Performance of the E-iMOD technique to detect a vulnerable plaque when the whole necrotic core is included in the endoluminal plaque domain. (a) Intravascular ultrasound image of plaque \#9 where red lines delimit necrotic core inclusion, lumen and external plaque contours. The blue contour delimits the endoluminal plaque domain. (b) Radial strain-elastogram and amplitude of the displacement vector applied on the intraplaque outer endoluminal boundary. (c) Spatial distribution of the normalized modified Sumi's transform (MST) criterion and the extracted inclusions contours $(n=20)$ obtained from the combined MST-DWS procedure. (d) Resulting plaque morphology and modulogram. 
regions deteriorated significantly from $4.93 \pm 0.68 \mathrm{kPa}$ and $784.72 \pm 15.97 \mathrm{kPa}$ when considering both components to $16.06 \pm 14.67 \mathrm{kPa}$ and $782.61 \pm 227.31 \mathrm{kPa}$ when considering only the radial displacement, instead of expected values of $5 \mathrm{kPa}$ and $800 \mathrm{kPa}$, respectively (results not shown).

\section{Performance of the E-iMOD method to detect VPs with} soft inclusions

Modulograms obtained for VPs with one necrotic core (plaques \#3, 9 and M1 to M3, Figs. 1 and 2) and two necrotic cores (plaques \#4 and 8, Figs. 3 and 4) are presented. Despite the fact that the necrotic cores were not entirely included in the endoluminal region of interest $\Omega_{\text {endo }}$, the E-iMOD technique was able to detect the structure (plaques \#3, M1 and M2, Fig. 1). While the Young's modulus of the fibrous region was accurately identified (computed mean value of $794.47 \pm 21.97 \mathrm{kPa}$ for a theoretical value equal to $800 \mathrm{kPa}$, Table 2), the stiffness of the soft inclusions was slightly overestimated (computed mean values of $5.67 \pm 0.82 \mathrm{kPa}$ instead $5 \mathrm{kPa}$, Table 2).

\section{Performance of the E-iMOD method to detect VPs with} calcified inclusions

For the highly heterogeneous VPs with calcified inclusions (plaques \#5 and 6, Figs. 5 and 6), the E-iMOD technique detected and differentiated the soft and hard inclusions. Figure 5 illustrates the abilities of the proposed E-iMOD technique to characterize a complex VP (plaque \#5) with adjacent soft and hard inclusions located between 2 and 4 o'clock and between 4 and 5 o'clock, respectively. The mean Young's modulus amplitude of the calcified inclusion was underestimated by a factor 1.5 (Table 2).
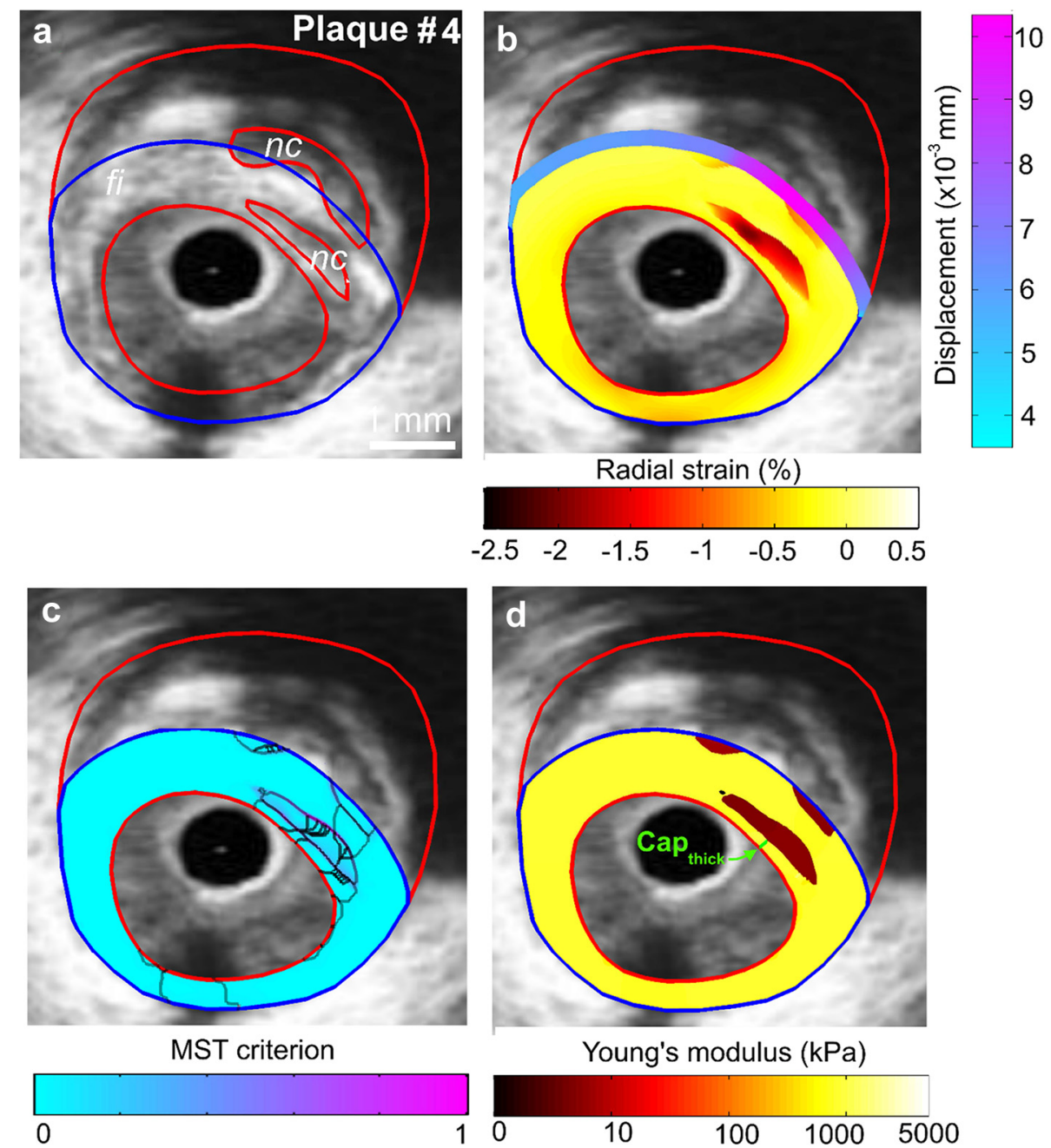

Fig. 3. Performance of the E-iMOD technique to detect a vulnerable plaque with two distant necrotic cores. (a) Intravascular ultrasound image of plaque \#4 with plaque constituents (red contours). (b) Radial strain-elastogram and displacement applied on the intraplaque outer endoluminal boundary. (c) Spatial distribution of the normalized MST criterion and extracted inclusions contours $(\mathrm{n}=40)$. (d) Computed modulogram. $\mathrm{nc}=$ necrotic core; fi $=$ fibrous region. 

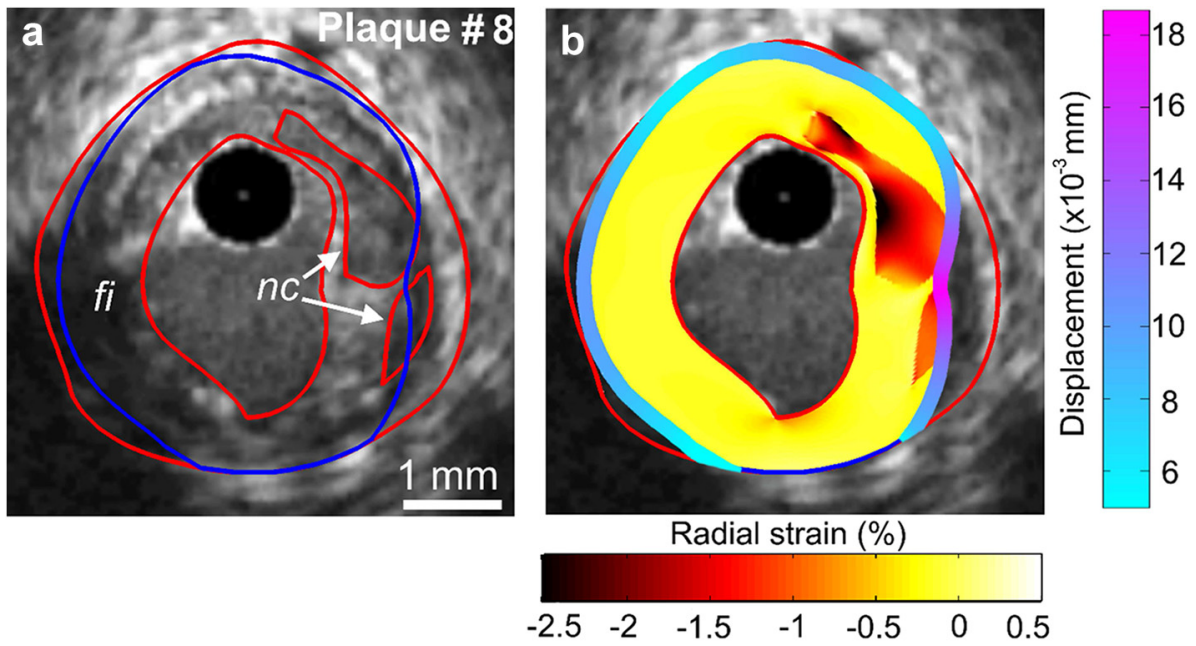

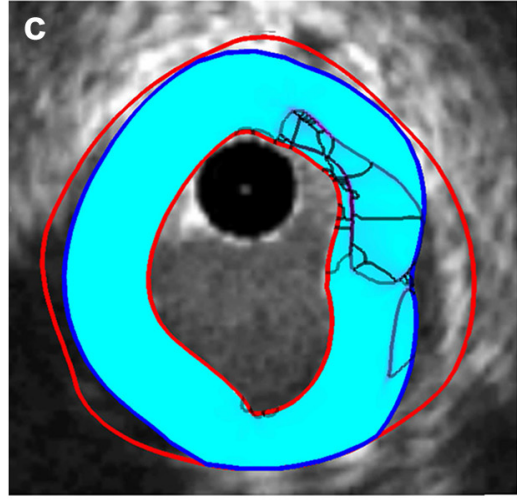

MST criterion

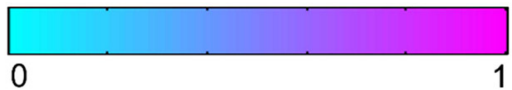

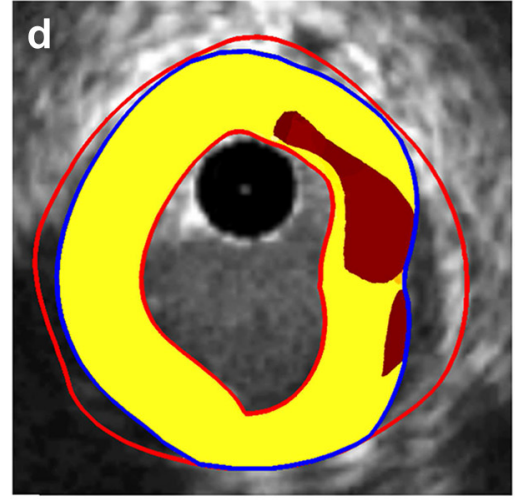

Young's modulus $(\mathrm{kPa})$

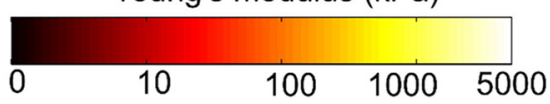

Fig. 4. Performance of the E-iMOD technique to detect a vulnerable plaque with two neighboring necrotic cores. (a) Intravascular ultrasound image of plaque \#8 with plaque constituents (red contours). (b) Radial strain-elastogram and displacement applied on the intraplaque outer endoluminal boundary. (c) Spatial distribution of the normalized MST criterion and extracted inclusions contours $(\mathrm{n}=40)$. (d) Computed modulogram. $\mathrm{nc}=$ necrotic core; fi $=$ fibrous region.

\section{Influence of the objective function and MST-DWS segmentation procedure on plaque elasticity reconstruction}

To test the performance of the proposed $\mathrm{RMS}_{\text {error }}$ function (i.e., with $\chi_{k=1.2 .3}=\varepsilon_{r r}, u_{r}, u_{\theta}$ in Eq. [6]) on elasticity map reconstructions, we compared the modulograms computed by using such objective function with those obtained when considering either only $\chi_{k=1,2}=\varepsilon_{r r}, u_{r}$ or only $\chi_{k=1}=\varepsilon_{r r}$ in the $\mathrm{RMS}_{\text {error }}$ formulation Eq. (6). By performing our simulations with real plaque morphologies, we found that the mean Young's moduli of all necrotic cores and fibrous regions varied slightly from $4.95 \pm 0.64 \mathrm{kPa}$ and $789.39 \pm 8.53 \mathrm{kPa}$ when considering the $R M S_{\text {error }}$ function with $\left(\varepsilon_{r r}, u_{r}, u_{\theta}\right)$ to $5.06 \pm 0.76 \mathrm{kPa}$ and $789.82 \pm$ $10.25 \mathrm{kPa}$ when considering the $R M S_{\text {error }}$ function with $\left(\varepsilon_{r r}, u_{r}\right)$ and to $4.93 \pm 0.68 \mathrm{kPa}$ and $784.72 \pm 15.97 \mathrm{kPa}$ when using the $R M S_{\text {error }}$ function with $\left(\varepsilon_{r r}\right)$, instead of expected values of $5 \mathrm{kPa}$ and $800 \mathrm{kPa}$, respectively (results not shown). However, by using the inclusions' contours extracted from the MST-DWS procedure, we found that the mean Young's moduli of all necrotic cores and fibrous regions varied from $5.67 \pm 0.82 \mathrm{kPa}$ and $794.47 \pm 21.97 \mathrm{kPa}$ when considering the $R M S_{\text {error }}\left(\varepsilon_{r r}, u_{r}, u_{\theta}\right)$ to $5.96 \pm 1.53 \mathrm{kPa}$ and $788.16 \pm$ $34.53 \mathrm{kPa}$ when considering the $R M S_{\text {error }}\left(\varepsilon_{r r}, u_{r}\right)$ and to $5.93 \pm 1.65 \mathrm{kPa}$ and $788.73 \pm 41.02 \mathrm{kPa}$ when using the $R M S_{\text {error }}\left(\varepsilon_{r r}\right)$, respectively (only results obtained with the objective function $R M S_{\text {error }}\left(\varepsilon_{r r}, u_{r}, u_{\theta}\right)$ are presented in Table 2). We can thus conclude that using only radial displacement and deformation, which are components that are easier to measure with real IVUS data sets, one could obtain acceptable modulograms. 
Table 2. Analysis of the performance of the proposed elasticity reconstruction method E-iMOD. Comparisons between computed and real plaque cap thickness $\left(\mathrm{Cap}_{\text {thick }}\right)$, necrotic core area in the endoluminal region of interest $\Omega_{\text {endo }}\left(\mathrm{Area}_{\text {core }}\right)$ and Young's moduli are presented. $\mathrm{E}_{\mathrm{core}}, \mathrm{E}_{\mathrm{fibrosis}}$ and $\mathrm{E}_{\mathrm{calcified}}$ are the Young's moduli of the necrotic core, fibrous region and calcified inclusion, respectively

\begin{tabular}{|c|c|c|c|c|c|c|c|c|c|}
\hline & \multirow{2}{*}{$\begin{array}{c}\begin{array}{c}\mathrm{E}_{\text {core }}(\mathrm{kPa}) \\
{[\mathrm{Real}=5 \mathrm{kPa}]}\end{array} \\
\text { Computed }\end{array}$} & \multicolumn{3}{|c|}{ Area $a_{\text {core }}\left(\mathrm{mm}^{2}\right)$} & \multirow{2}{*}{$\frac{\begin{array}{c}\mathrm{E}_{\mathrm{fibrosis}}(\mathrm{kPa}) \\
{[\mathrm{Real}=800 \mathrm{kPa}]}\end{array}}{\text { Computed }}$} & \multirow{2}{*}{$\begin{array}{c}\begin{array}{c}\mathrm{E}_{\text {calcified }}(\mathrm{kPa}) \\
{[\mathrm{Real}=5 \mathrm{kPa}]}\end{array} \\
\text { Computed }\end{array}$} & \multicolumn{3}{|c|}{ Cap thickness $(\mu \mathrm{m})$} \\
\hline & & Computed & Real & Relative error & & & Computed & Real & Relative error \\
\hline Plaque \#2 & $5.03 \pm 0.08$ & 1.35 & 1.36 & $-0.7 \%$ & $798.07 \pm 25.53$ & - & 91 & 93 & $-2.7 \%$ \\
\hline Plaque \#3 & 5.67 & 1.64 & 1.65 & $-0.6 \%$ & $805.30 \pm 83.11$ & - & 84 & 100 & $-16.2 \%$ \\
\hline Plaque \#M1 & $6.05 \pm 0.57$ & 1.42 & 1.43 & $-0.7 \%$ & $825.75 \pm 99.48$ & - & 160 & 181 & $-11.6 \%$ \\
\hline Plaque \#M2 & 7.65 & 1.02 & 1.00 & $2.0 \%$ & $792.50 \pm 79.86$ & - & 360 & 363 & $-0.8 \%$ \\
\hline Plaque \#M3 & - & - & - & - & $792.25 \pm 16.88$ & - & - & 1304 & - \\
\hline \multicolumn{10}{|l|}{ Plaque \#4 } \\
\hline Core 1 & $4.99 \pm 0.28$ & 0.45 & 0.51 & $-11.8 \%$ & $791.67 \pm 38.96$ & - & 147 & 162 & $-8.7 \%$ \\
\hline Core 2 & $6.09 \pm 1.44$ & 0.23 & 0.22 & $4.5 \%$ & & & & & \\
\hline Plaque \#5 & $6.32 \pm 0.93$ & 0.94 & 0.99 & $-5.1 \%$ & $820.14 \pm 180.43$ & $2180 \pm 500$ & 68 & 41 & $64.6 \%$ \\
\hline Plaque \#6 & $5.15 \pm 1.54$ & 2.72 & 2.92 & $-6.8 \%$ & $752.57 \pm 496.98$ & $4430 \pm 29.1$ & 32 & 62 & $-47.6 \%$ \\
\hline \multicolumn{10}{|l|}{ Plaque \#8 } \\
\hline Core 1 & $5.26 \pm 0.33$ & 1.31 & 1.32 & $-0.8 \%$ & $766.66 \pm 40.96$ & - & 49 & 52 & $-6.1 \%$ \\
\hline Core 2 & 4.87 & 0.22 & 0.23 & $-4.3 \%$ & & & & & \\
\hline Plaque 9 & $5.33 \pm 0.71$ & 0.86 & 0.87 & $-1.1 \%$ & $799.82 \pm 13.55$ & - & 57 & 50 & $14.2 \%$ \\
\hline Mean \pm SD & $5.67 \pm 0.82$ & - & - & - & $794.47 \pm 21.97$ & $3301.28 \pm 1591.14$ & - & - & - \\
\hline
\end{tabular}

$\mathrm{SD}=$ standard deviation

\section{Performance of the E-iMOD method to quantify cap thickness amplitudes}

Based on the results obtained on our entire population, we show that $\mathrm{Cap}_{\text {thick }}$ amplitudes are with minimal and maximal relative errors close to $-0.8 \%$ for plaque \#M2 (corresponding to a computed Cap thick of $360 \mu \mathrm{m}$ for a theoretical value equal to $363 \mu \mathrm{m}$, see Fig. 1 and Table 2) and $+64.6 \%$ for plaque \#5 (corresponding to a computed Cap thick $_{\text {of }} 68 \mu \mathrm{m}$ instead of $41 \mu \mathrm{m}$, see Fig. 5d and Table 2), respectively. Figure 1 illustrates the performance of E-iMOD algorithm to detect vulner-

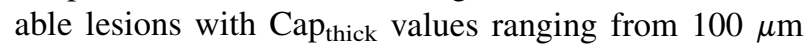
to $363 \mu \mathrm{m}$, respectively.

\section{Influence of noise on plaque elasticity reconstruction}

The vulnerable atherosclerotic plaque \#2 with a $\mathrm{Cap}_{\text {thick }}$ and necrotic core area $\left(\mathrm{Area}_{\text {core }}\right)$ equal to 93 $\mu \mathrm{m}$ and $1.36 \mathrm{~mm}^{2}$, respectively, was used to study the influence of noise on Young's modulus and plaque morphology reconstructions (Figs. 7 and 8). Table 3 provides the correspondence between the imposed white noise (controlled by the parameter $\beta, 1 \leq \beta \leq 6$ ) and the signal-to-noise ratio (SNR, in $\mathrm{dB}$ ). Figure 7 presents the sensitivity of the E-iMOD algorithm when increasing the white noise level. The performed simulations showed that the E-iMOD method was able to highlight the VP morphology when introducing significant white noise on both radial strain and displacement fields was introduced $\left(\beta=6\right.$, Fig. 7). The Capthick and Area $\mathrm{c}_{\text {core }}$ amplitudes were under-estimated and vary from $87.19 \pm 7.51$ $\mu \mathrm{m}$ and $1.32 \pm 0.03 \mathrm{~mm}^{2}$ with a white noise of $\beta=1$ to $82.75 \pm 5.63 \mu \mathrm{m}$ and $1.18 \pm 0.28 \mathrm{~mm}^{2}$ with triple white noise $(\beta=6)$, respectively (Fig. 8a, d). The EiMOD method reasonably identified mean Young's moduli of the necrotic core and fibrous region with both maximal relative error lower than 20\% (Fig. 8b, c).

\section{DISCUSSION}

The SNR acquired with the HD-IVUS systems may allow a better estimation of the intraplaque radial strain distribution in the endoluminal plaque region than in the adventitial region (i.e., deeper tissue; Kobayashi et al. 2014; Waters et al. 2011). Therefore, in the current study, the native iMOD approach was successfully revisited and extended to permit the elasticity reconstruction of the endoluminal atherosclerotic region only, based on the reliability of the strain field measured in such zone.

\section{Is the elasticity-palpogram sufficient to predict the degree of instability of a VP?}

Knowing that one of the critical keys in detection of VPs is the quantification of their mechanical properties, Céspedes et al. (2000) proposed an elasticitypalpography (E-P) approach. This technique estimates the apparent stiffness of the endoluminal plaque region. Although the wall stiffness quantification was computationally very fast, it suffers from major limitations because it was developed for homogeneous, isotropic, circular and concentric VPs. Therefore, Deleaval et al. (2013) revisited and extended the theoretical framework of the E-P approach by considering the anatomical shape of the atherosclerotic lesion. They demonstrated that the 

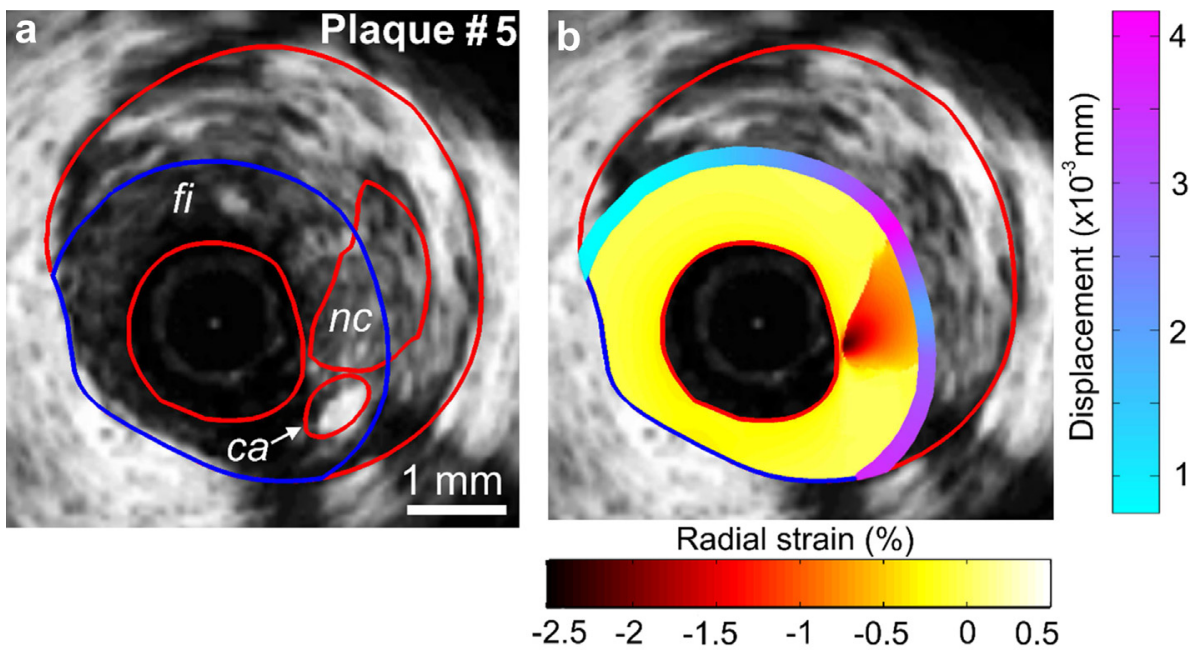

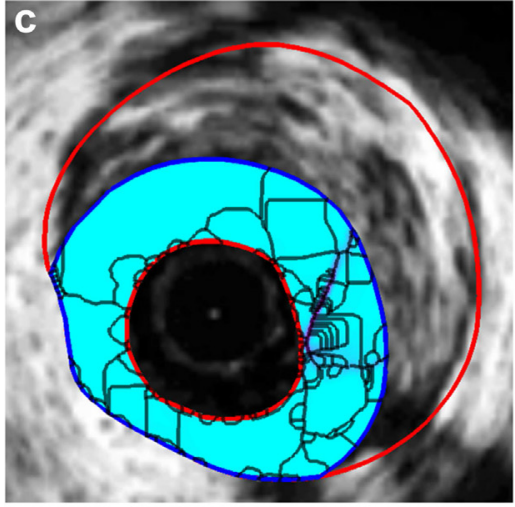

MST criterion

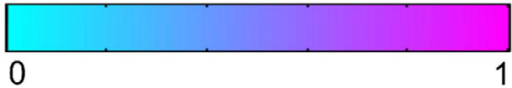

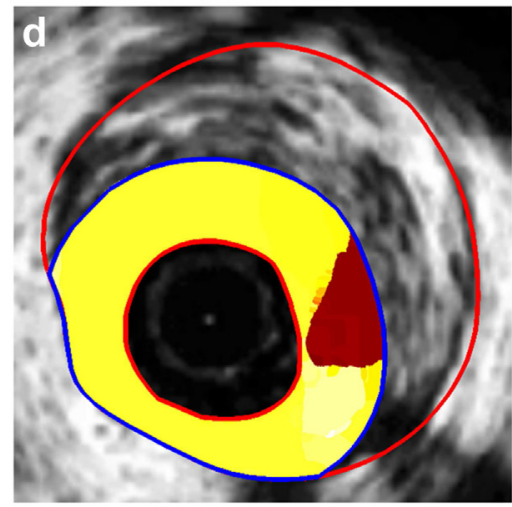

Young's modulus $(\mathrm{kPa})$

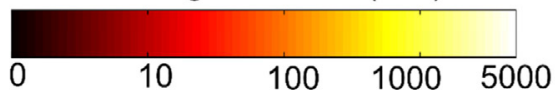

Fig. 5. Performance of the proposed E-iMOD technique to detect a vulnerable plaque with a small, calcified inclusion adjacent to a necrotic core. (a) Intravascular ultrasound image of plaque \#5 with plaque constituents (red contours). (b) Radial strain-elastogram and displacement applied on the intraplaque outer endoluminal boundary. (c) Spatial distribution of the normalized modified Sumi's transform criterion and extracted inclusions contours $(\mathrm{n}=150)$. (d) Computed modulogram. $\mathrm{nc}=$ necrotic core; $\mathrm{fi}=$ fibrous region; $\mathrm{ca}=$ calcified inclusion

revisited E-P (RE-P) technique was sufficient to detect and identify plaques \#2, 3, 5 and 6 considered in the present study (see palpograms presented on Figs. 2, 3, 5 and 6 of Deleaval et al. 2013). However, The RE-P technique does not permit direct measurements of the key geometrical VP determinants as the fibrous cap thickness, plaque morphology, and therefore cannot quantify the PCS.

\section{Is the proposed E-iMOD algorithm adapted to detect and diagnose the degree of instability of a VP?}

From the current clinical data, the E-iMOD algorithm is able to detect small Cap thick $_{\text {amplitudes, necrotic }}$ core morphology and mechanical properties in the endoluminal region. The geometrical plaque features are known to be the major clinical criteria used by interventional cardiologists for predicting plaque vulnerability.
For a VP with a potential high degree of vulnerability (plaque \#6, which had a Cap thick of $62 \mu \mathrm{m}$ ), the proposed algorithm is able to detect the Cap thick $_{\text {(estimated }}$ $\mathrm{Cap}_{\text {thick }}=32 \mu \mathrm{m}$, see Fig. 6 and Table 2). This performance was linked with the radial resolution of $15.4 \mu \mathrm{m}$ used in our polar mesh grid, which was adapted to the resolution of the Lagrangian Speckle Model Estimator method developed by Maurice et al. (2007). However, the lower Cap thick that can be detected with the IVUS system will be within the limit of the expected resolution obtained with the IVUS catheter (i.e., close to $120 \mu \mathrm{m}$ for a 20-MHz catheter, $90 \mu \mathrm{m}$ for a $40-\mathrm{MHz}$ and $40 \mu \mathrm{m}$ for HD-IVUS $>60 \mathrm{MHz}$; Righetti et al. 2002). Moreover, note that quantifying PCS amplitude in vivo (and then diagnosing the degree of instability of a VP) could be now performed as the E-iMOD method allows us to 

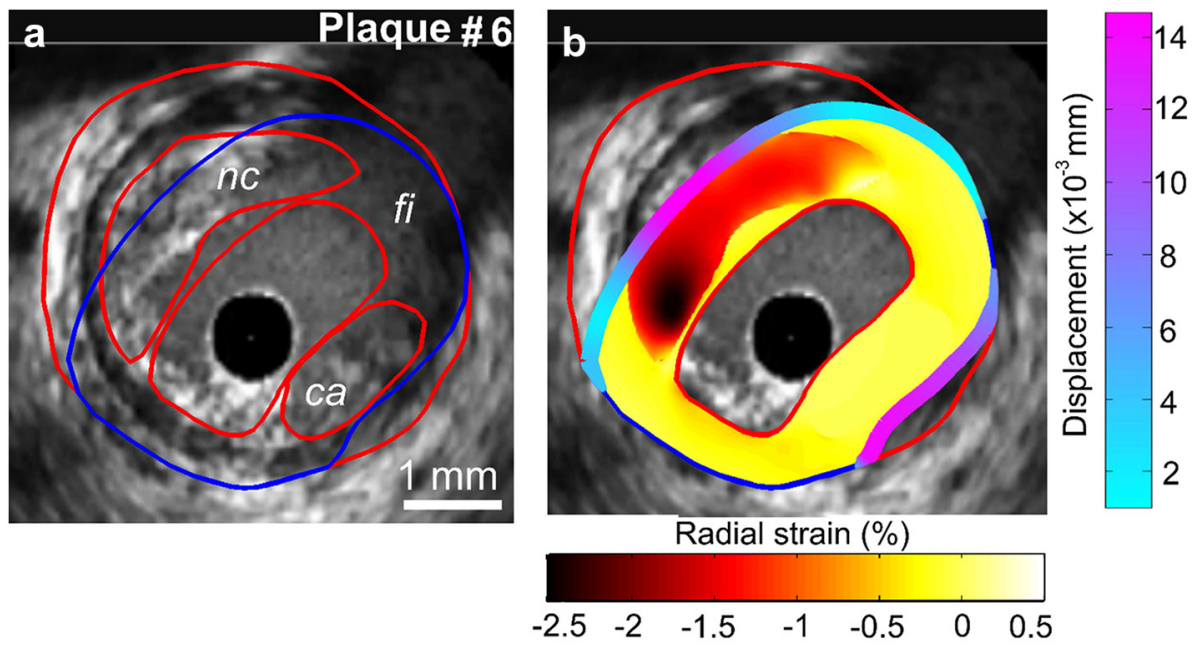

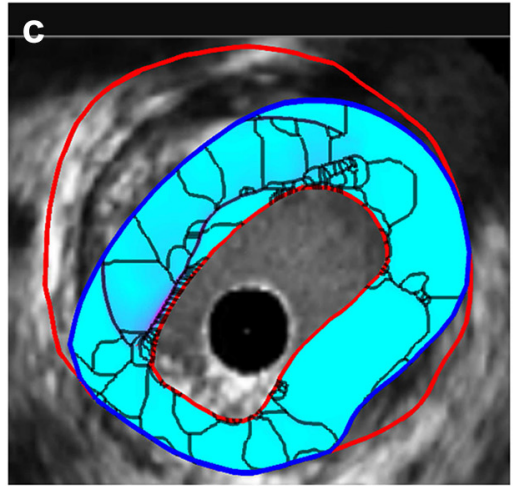

MST criterion

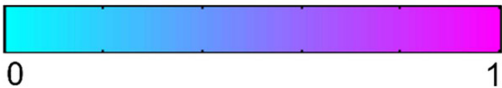

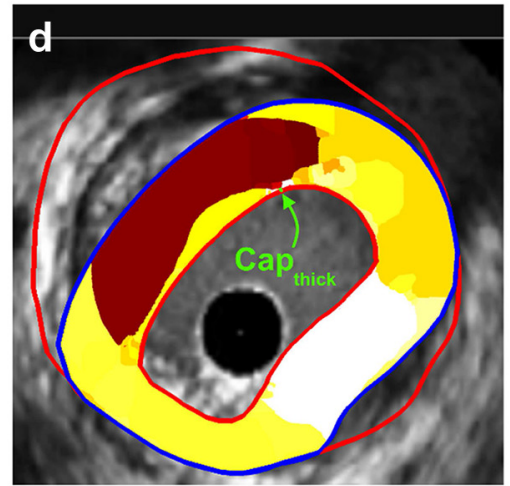

Young's modulus $(\mathrm{kPa})$

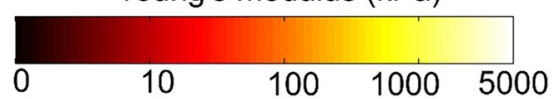

Fig. 6. Performance of the proposed E-iMOD technique to detect a vulnerable plaque with a large calcified inclusion. (a) Intravascular ultrasound image of plaque \#6 with plaque contours. (b) Radial strain-elastogram and displacement applied on the intraplaque outer endoluminal boundary. (c) Spatial distribution of the normalized modified Sumi's transform criterion and extracted inclusions contours $(n=150)$. (d) Computed modulogram. $n c=$ necrotic core; fi $=$ fibrous region; $\mathrm{ca}=$ calcified inclusion.

extract both the atherosclerotic plaque morphology and the mechanical properties of all plaque components.

\section{Is the proposed E-iMOD algorithm adapted to detect} calcified inclusions?

We also investigated the performance of our algorithm in detecting large calcified inclusions within the endoluminal region of the heterogeneous plaques (Figs. 5 and 6). Our results demonstrated that the estimated Young's moduli were significantly underestimated (up to a factor 1.50) but remained high enough as to reveal the presence of rigid inclusions (Table 2). This is mainly due to the noise that stands in calcified regions, which are sites of small strain amplitudes. Similar effects were observed by Le Floc'h et al. (2009) when using the elasticity technique iMOD. Notice that the detection of calcified areas located in the thin fibrous cap is of clinical importance as Cardoso et al. (2014) demonstrated that calcified inclusions significantly affect the plaque's stability.

The proposed E-iMOD algorithm may be used to characterize the nonlinear mechanical properties of the atherosclerotic lesion

Most biological tissues, including the coronary artery wall, have a nonlinear stress-strain behavior (Holzapfel et al. 2005). In the proposed E-iMOD model, the elastic behaviors of the plaque constituents were assumed to be linear. This assumption remains acceptable as strain fields measured between two successive frames of an IVUS sequence (30 frames/sec) were used (Le Floc'h et al. 2012). Such a technique may be used to incrementally characterize in vivo the nonlinear mechanical responses of atherosclerotic plaque components 


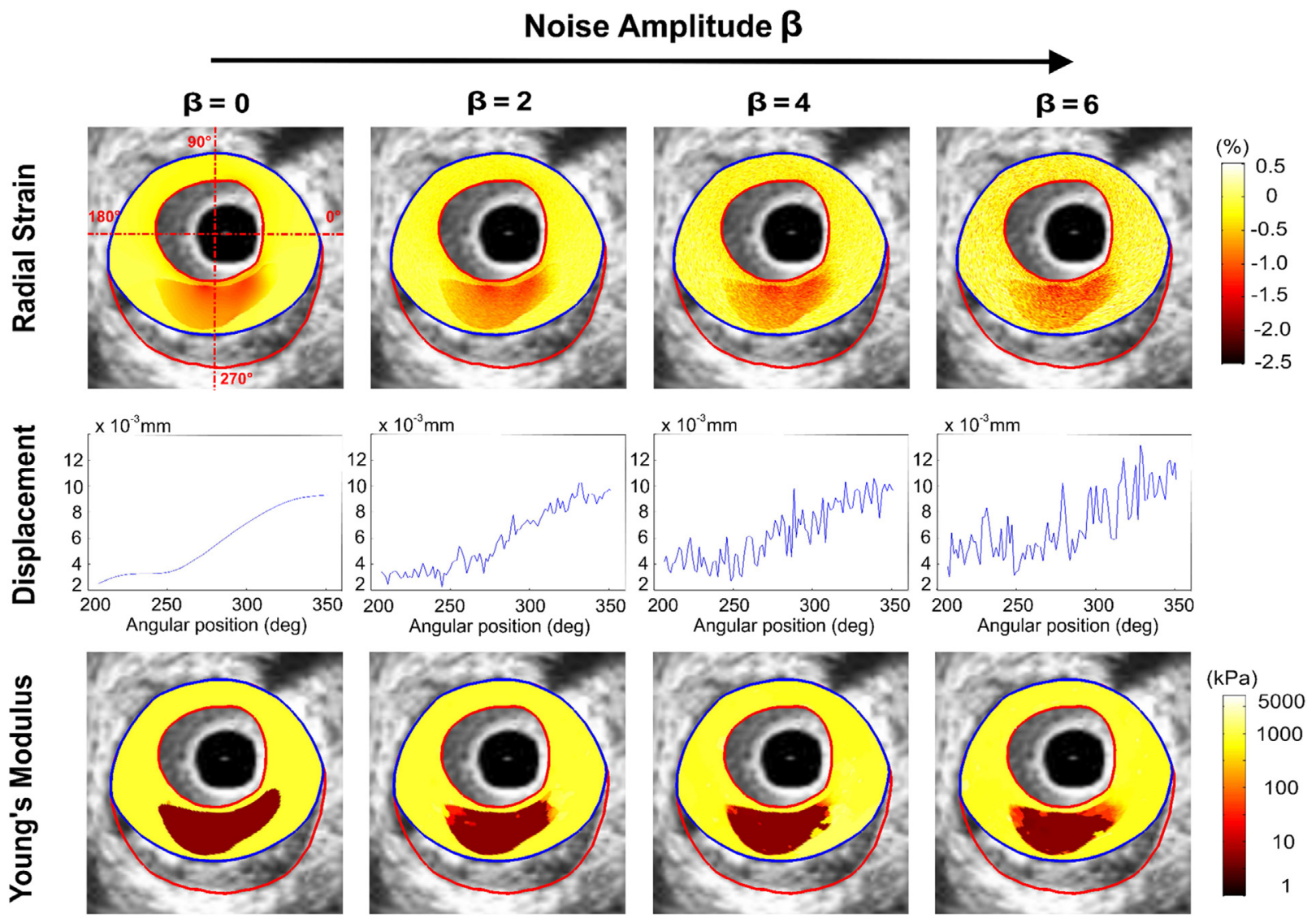

Fig. 7. Results of the sensitivity analysis performed to investigate the influence of the white noise added to the strain and displacement fields on the reconstructed modulograms. Plaque \#2 was used for this study. Row 1: Input radial strain fields obtained with different levels of white noise $(\beta=2,4$ and 6). Row 2: Input displacement fields applied on the intraplaque outer endoluminal boundary and obtained with different levels of white noise ( $\beta=2,4$ and 6$)$. Row 3: Resulting plaque morphologies and modulograms. For all these simulations, the numbers of extracted inclusions contours were equal to 20.

occurring during the cardiac cycle. This has been successfully tested and validated using an in vitro experimental study performed by our group with the native iMOD algorithm on vessel phantoms (Le Floc'h et al. 2010).

\section{Study limitations}

Although this technique presents original and potentially promising concepts for IVUS identification and characterization of atherosclerotic lesions, several limitations deserve to be pointed out at this stage.

The first one is that the proposed IVUS-elasticity reconstruction technique E-iMOD has been tested on a limited number of in vivo plaque geometries $(\mathrm{n}=7)$.

A second study limitation was that all plaque constituents, including the arterial wall, were assumed to be isotropic. The anisotropic mechanical properties of the normal artery components (i.e., intima, media and adventitia) have been well described in the elegant experimental and theoretical works of Holzapfel et al. (2005) for human coronaries. However, a sensitivity study with regard to material properties (comparing the mechanical responses of atherosclerotic lesions when the plaque constituents were assumed to be anisotropic or isotropic) has been performed by Kamm's group (Williamson et al. 2003). They found that either isotropic or anisotropic models provide similar results for the prediction of PCS location in diseased arteries with a PCS underestimation close to $30 \%$ when considering isotropic media. Moreover, this point should be seen in the light of our aim, which was to develop new IVUS-based imaging techniques that (i) automatically identify the contours of all components (including the necrotic cores and calcified inclusions) of the plaque, (ii) highlight morphology of lesions, (iii) quantify the cap thickness amplitude and (iv) permit estimation of the mean homogenized (or apparent) isotropic Young's moduli of the necrotic core and all other plaque constituents. However, more sophisticated anisotropic elasticity in vivo reconstruction methods deserve to be investigated in a future study to overcome such limitations. 


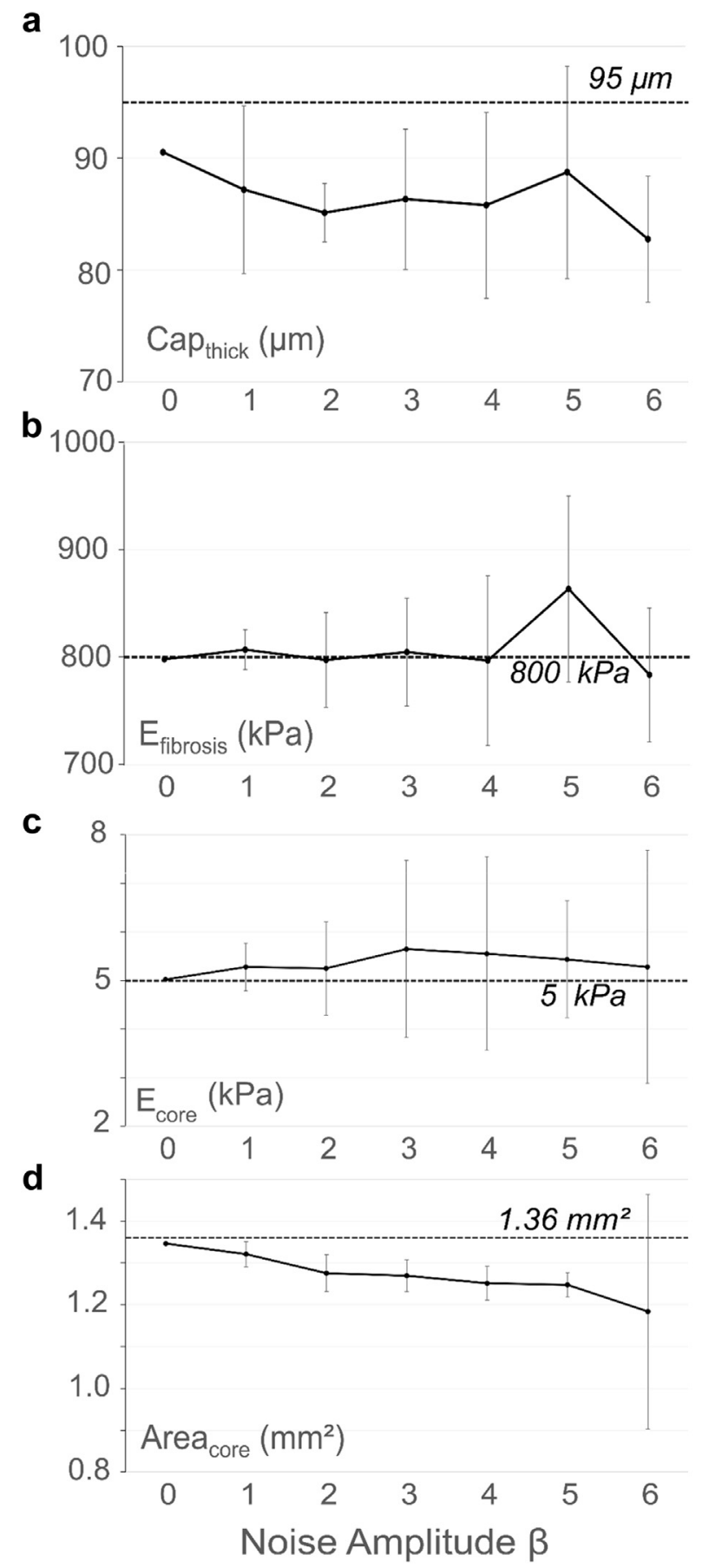

Fig. 8. Influence of white noise amplitude on estimations of cap thickness $\left(\mathrm{Cap}_{\text {thick }}\right)$, Young's moduli of the necrotic core $\left(\mathrm{E}_{\text {core }}\right)$ and fibrosis $\left(\mathrm{E}_{\text {fibrosis }}\right)$ and necrotic core area $\left(\right.$ Area $\left.\mathrm{a}_{\text {core }}\right)$. Plaque \#2 was used for this investigation. Knowing that the noise was spatially randomly distributed, we performed 20 computations for each amplitude of noise $\beta$ and present the mean values \pm the standard deviations. The dashed lines indicate the values used for the forward problem. (a) Influence of noise on $\mathrm{Cap}_{\text {thick }}$ estimation. (b) Influence of noise on $\mathrm{E}_{\text {fibrosis }}$ estimation. (c) Influence of noise on $\mathrm{E}_{\text {core }}$ estimation. (d) Influence of noise on Area $_{\text {core }}$ estimation.
Table 3. Correspondence between the imposed levels of white noise (i.e. $\beta$ ) and the signal-to-noise ratio (SNR) amplitudes. For each level of noise $\beta, 20$ computations in which the noise was spatially randomly distributed were performed. Mean SNR and standard deviation values of input radial strain $\left(\varepsilon_{r r}\right)$, radial displacement $\left(u_{r}\right)$ and circumferential displacement $\left(u_{\theta}\right)$ are given. Plaque \#2 was used for this investigation

\begin{tabular}{lrrr}
\hline & \multicolumn{3}{c}{ SNR $(\mathrm{dB})$} \\
\cline { 2 - 4 }$\beta$ & \multicolumn{1}{c}{$\varepsilon_{r r}$} & \multicolumn{1}{c}{$u_{r}$} & \multicolumn{1}{c}{$u_{\theta}$} \\
\hline 1 & $18.74 \pm 0.06$ & $20.33 \pm 0.04$ & $16.57 \pm 0.04$ \\
2 & $12.97 \pm 0.05$ & $14.31 \pm 0.05$ & $10.53 \pm 0.04$ \\
3 & $9.45 \pm 0.06$ & $10.79 \pm 0.04$ & $7.00 \pm 0.05$ \\
4 & $6.93 \pm 0.08$ & $8.24 \pm 0.06$ & $4.53 \pm 0.07$ \\
5 & $4.98 \pm 0.05$ & $6.36 \pm 0.03$ & $2.56 \pm 0.03$ \\
6 & $3.42 \pm 0.06$ & $4.76 \pm 0.04$ & $0.99 \pm 0.04$ \\
\hline
\end{tabular}

A third limitation of this study was that the full 3-D structures of the atherosclerotic lesions have not been considered in this study because only 2-D IVUS sequences are acquired in routine imaging during the clinical protocol. Therefore, the FE simulations were conducted under plane strain assumption. This assumption is reasonable insofar as (i) plaque length is large with regard to the radial dimension of the artery and (ii) neighboring cross-sectional morphologies remain similar (Ohayon et al. 2005).

\section{Potential clinical implications}

Our group (Finet et al. 2004) demonstrated that a very slight change in the Young's moduli of plaque constituents, namely the hardening of the soft necrotic core, can shift a VP from instability to stability. This finding is in agreement with several other studies conducted to analyze the effects of statins and angiotensin-converting enzyme inhibitors on structural variation in the fibrous cap and necrotic core, revealing an enhancement in plaque stability (Abela et al. 2011; Libby et al. 2002; Nie et al. 2014; Nozue et al. 2012; Yla-Herttuala et al. 2011). Due to its performance and accuracy, the proposed E-iMOD elasticity imaging technique could provide an original approach to analyze the evolution of the mechanical properties of atherosclerotic plaques during drug therapies.

Acknowledgments-This research was supported by a joint international program of the Agence Nationale de la Recherche (ANR) grant MELANII project \#09-BLAN-0423 and of the of the Natural Sciences and Engineering Research Council of Canada (NSERC) strategic grant \#STPGP-381136-09. Partial support was provided by NSERC discovery grant \#138570-11. The authors thank Dr. Flavien Deleaval (TIMC Laboratory, Grenoble) for computational support and Dr Saami K. Yazdani (Department of Mechanical Engineering, University of South Alabama, Mobile, AL, USA) for helpful discussions. Antoine Tacheau held a doctoral fellowship from University Grenoble-Alpes, France (AGIR 2013). 


\section{REFERENCES}

Abela GS, Vedre A, Janoudi A, Huang R, Durga S, Tamhane U. Effect of statins on cholesterol crystallization and atherosclerotic plaque stabilization. Am J Cardiol 2011;107:1710-1717.

Baldewsing RA, Mastik F, Schaar JA, Serruys PW, van der Steen AF. Robustness of reconstructing the Young's modulus distribution of vulnerable atherosclerotic plaques using a parametric plaque model. Ultrasound Med Biol 2005;31:1631-1645.

Baldewsing RA, Danilouchkine MG, Mastik F, Schaar JA, Serruys PW, van der Steen AF. An inverse method for imaging the local elasticity of atherosclerotic coronary plaques. IEEE Trans Inf Technol Biomed 2008; 12:277-289.

Bouvier A, Deleaval F, Doyley MM, Yazdani SK, Finet G, Le Floc'h S, Cloutier G, Pettigrew RI, Ohayon J. A direct vulnerable atherosclerotic plaque elasticity reconstruction method based on an original material-finite element formulation: theoretical framework. Phys Med Biol 2013;58:8457-8476.

Broisat A, Toczek J, Mesnier N, Tracqui P, Ghezzi C, Ohayon J, Riou L. Assessing the low levels of mechanical stress in aortic atherosclerosis lesions from ApoE-/-mouse. Arterioscler Thromb Vasc Biol 2011:31:1007-1010.

Cardoso L, Kelly-Arnold A, Maldonado N, Laudier D, Weinbaum S. Effect of tissue properties, shape and orientation of microcalcifications on vulnerable cap stability using different hyperelastic constitutive models. J Biomech 2014:47:870-877.

Cespedes I, Ophir J, Ponnekanti H, Maklad N. Elastography: elasticity imaging using ultrasound with application to muscle and breast in vivo. Ultrason Imaging 1993;15:73-88.

Céspedes EI, de Korte CL, van der Steen AF. Intraluminal ultrasonic palpation: assessment of local and cross-sectional tissue stiffness. Ultrasound Med Biol 2000;26:385-396.

Cheng GC, Loree HM, Kamm RD, Fishbein MC, Lee RT. Distribution of circumferential stress in ruptured and stable atherosclerotic lesions. A structural analysis with histopathological correlation. Circulation 1993;87:1179-1187.

de Korte CL, Carlier SG, Mastik F, Doyley MM, van der Steen AF, Serruys PW, Bom N. Morphological and mechanical information of coronary arteries obtained with intravascular elastography; feasibility study in vivo. Eur Heart J 2002;23:405-413.

Deleaval F, Bouvier A, Finet G, Cloutier G, Yazdani SK, Le Floc'h S, Clarysse P, Pettigrew RI, Ohayon J. The intravascular ultrasound elasticity-palpography technique revisited: a reliable tool for the in vivo detection of vulnerable coronary atherosclerotic plaques. Ultrasound Med Biol 2013:39:1469-1481.

Di Mario C, Gorge G, Peters R, Kearney P, Pinto F, Hausmann D, von Birgelen C, Colombo A, Mudra H, Roelandt J, Erbel R. Clinical application and image interpretation in intracoronary ultrasound. Study Group on Intracoronary Imaging of the Working Group of Coronary Circulation and of the Subgroup on Intravascular Ultrasound of the Working Group of Echocardiography of the European Society of Cardiology. Eur Heart J 1998;19:207-229.

Doyley MM, Mastik F, de Korte CL, Carlier SG, Cespedes EI, Serruys PW, Bom N, van der Steen AF. Advancing intravascular ultrasonic palpation toward clinical applications. Ultrasound Med Biol 2001;27:1471-1480.

Doyley MM. Model-based elastography: a survey of approaches to the inverse elasticity problem. Phys Med Biol 2012;57:R35-R73.

Finet G, Ohayon J, Rioufol G. Biomechanical interaction between cap thickness, lipid core composition and blood pressure in vulnerable coronary plaque: Impact on stability or instability. Coron Artery Dis 2004; $15: 13-20$.

Fleg JL, Stone GW, Fayad ZA, Granada JF, Hatsukami TS, Kolodgie FD, Ohayon J, Pettigrew R, Sabatine MS, Tearney GJ, Waxman S, Domanski MJ, Srinivas PR, Narula J. Detection of high-risk atherosclerotic plaque: Report of the NHLBI Working Group on Current Status and Future Directions. JACC Cardiovasc Imaging 2012;5:941-955.

Go AS, Mozaffarian D, Roger VL, Benjamin EJ, Berry JD, Borden WB, Bravata DM, Dai S, Ford ES, Fox CS, Franco S, Fullerton HJ, Gillespie C, Hailpern SM, Heit JA, Howard VJ, Huffman MD, Kissela BM, Kittner SJ, Lackland DT, Lichtman JH, Lisabeth LD,
Magid D, Marcus GM, Marelli A, Matchar DB, McGuire DK, Mohler ER, Moy CS, Mussolino ME, Nichol G, Paynter NP, Schreiner PJ, Sorlie PD, Stein J, Turan TN, Virani SS, Wong ND, Woo D, Turner MB. Executive summary: heart disease and stroke statistics-2013 update: a report from the American Heart Association. Circulation 2013;127:143-152.

Holzapfel GA, Sommer G, Gasser CT, Regitnig P. Determination of layer-specific mechanical properties of human coronary arteries with nonatherosclerotic intimal thickening and related constitutive modeling. Am J Physiol Heart Circ Physiol 2005;289: H2048-H2058.

Keshavarz-Motamed Z, Saijo Y, Majdouline Y, Riou L, Ohayon J, Cloutier G. Coronary artery atherectomy reduces plaque shear strains: An endovascular elastography imaging study. Atherosclerosis 2014;235:140-149.

Kobayashi Y, Kitahara H, Tanaka S, Nakagawa K, Okada K, Otagiri K, Yock P, Fitzgerald P, Ikeno F, Honda Y. TCT-363 precision of a novel high-definition $60 \mathrm{MHz}$ IVUS in quantitative measurement: comparison with conventional 40MHz IVUS and optical coherence tomography. J Am Coll Cardiol 2014;64:B105-B106.

Le Floc'h S, Ohayon J, Tracqui P, Finet G, Gharib AM, Maurice RL, Cloutier G, Pettigrew RI. Vulnerable atherosclerotic plaque elasticity reconstruction based on a segmentation-driven optimization procedure using strain measurements: theoretical framework. IEEE Trans Med Imaging 2009;28:1126-1137.

Le Floc'h S, Cloutier G, Finet G, Tracqui P, Pettigrew RI, Ohayon J. On the potential of a new IVUS elasticity modulus imaging approach for detecting vulnerable atherosclerotic coronary plaques: in vitro vessel phantom study. Phys Med Biol 2010;55:5701-5721.

Le Floc'h S, Cloutier G, Saijo Y, Finet G, Yazdani SK, Deleaval F, Rioufol G, Pettigrew RI, Ohayon J. A four-criterion selection procedure for atherosclerotic plaque elasticity reconstruction based on in vivo coronary intravascular ultrasound radial strain sequences. Ultrasound Med Biol 2012;38:2084-2097.

Libby P. Current concepts of the pathogenesis of the acute coronary syndromes. Circulation 2001;104:365-372.

Libby P, Ridker PM, Maseri A. Inflammation and atherosclerosis. Circulation 2002;105:1135-1143.

Lloyd-Jones D, Adams RJ, Brown TM, Carnethon M, Dai S, De Simone G, Ferguson TB, Ford E, Furie K, Gillespie C, Go A, Greenlund K, Haase N, Hailpern S, Ho PM, Howard V, Kissela B, Kittner S, Lackland D, Lisabeth L, Marelli A, McDermott MM, Meigs J, Mozaffarian D, Mussolino M, Nichol G, Roger VL, Rosamond W, Sacco R, Sorlie P, Stafford R, Thom T, Wasserthiel-Smoller S, Wong ND, Wylie-Rosett J. Executive summary: heart disease and stroke statistics-2010 update: a report from the American Heart Association. Circulation 2010;121: 948-954.

Loree HM, Kamm RD, Stringfellow RG, Lee RT. Effects of fibrous cap thickness on peak circumferential stress in model atherosclerotic vessels. Circ Res 1992;71:850-858.

Magnoni M, Ammirati E, Camici PG. Non-invasive molecular imaging of vulnerable atherosclerotic plaques. J Cardiol 2015;65:261-269.

Majdouline Y, Ohayon J, Keshavarz-Motamed Z, Roy Cardinal MH, Garcia D, Allard L, Lerouge S, Soulez G, Cloutier G. Endovascular shear strain elastography can detect and characterize the severity of atherosclerotic plaques: in vitro and in vivo validations. Ultrasound Med Biol 2014:40:890-903.

Maurice RL, Ohayon J, Finet G, Cloutier G. Adapting the Lagrangian speckle model estimator for endovascular elastography: theory and validation with simulated radio-frequency data. J Acoust Soc Am 2004;116:1276-1286.

Maurice RL, Fromageau J, Brusseau E, Finet G, Rioufol G, Cloutier G. On the potential of the lagrangian estimator for endovascular ultrasound elastography: in vivo human coronary artery study. Ultrasound Med Biol 2007;33:1199-1205.

Nie P, Li D, Hu L, Jin S, Yu Y, Cai Z, Shao Q, Shen J, Yi J, Xiao H, Shen L, He B. Atorvastatin improves plaque stability in ApoEknockout mice by regulating chemokines and chemokine receptors. PLoS One 2014;9:e97009.

Nozue T, Yamamoto S, Tohyama S, Umezawa S, Kunishima T, Sato A, Miyake S, Takeyama Y, Morino Y, Yamauchi T, Muramatsu T, 
Hibi K, Sozu T, Terashima M, Michishita I. Statin treatment for coronary artery plaque composition based on intravascular ultrasound radiofrequency data analysis. Am Heart J 2012;163:191-199.e1.

Ohayon J, Teppaz P, Finet G, Rioufol G. In-vivo prediction of human coronary plaque rupture location using intravascular ultrasound and the finite element method. Coron Artery Dis 2001;12:655-663.

Ohayon J, Finet G, Treyve F, Rioufol G, Dubreuil O. A threedimensional finite element analysis of stress distribution in a coronary atherosclerotic plaque: in-vivo prediction of plaque rupture location. In: Payan Y, (ed). Biomechanics applied to computer assisted surgery. Trivandrum, India: Research Signpost Publisher; 2005.

Ohayon J, Finet G, Gharib AM, Herzka DA, Tracqui P, Heroux J, Rioufol G, Kotys MS, Elagha A, Pettigrew RI. Necrotic core thickness and positive arterial remodeling index: emergent biomechanical factors for evaluating the risk of plaque rupture. Am J Physiol Heart Circ Physiol 2008;295:H717-H727.

Ohayon J, Finet G, Le Floc'h S, Cloutier G, Gharib AM, Heroux J, Pettigrew RI. Biomechanics of atherosclerotic plaque: sites, stability and in vivo elasticity modeling. Ann Biomed Eng 2014;42:269-279.

Ophir J, Cespedes I, Ponnekanti H, Yazdi Y, Li X. Elastography: a quantitative method for imaging the elasticity of biological tissues. Ultrason Imaging 1991;13:111-134.

Richards MS, Doyley MM. Investigating the impact of spatial priors on the performance of model-based IVUS elastography. Phys Med Biol 2011;56:7223-7246.

Richards MS, Doyley MM. Non-rigid image registration based strain estimator for intravascular ultrasound elastography. Ultrasound Med Biol 2013;39:515-533.

Righetti R, Ophir J, Ktonas P. Axial resolution in elastography. Ultrasound Med Biol 2002;28:101-113.

Riou LM, Broisat A, Ghezzi C, Finet G, Rioufol G, Gharib AM, Pettigrew RI, Ohayon J. Effects of mechanical properties and atherosclerotic artery size on biomechanical plaque disruption mouse versus human. J Biomech 2014;47:765-772.

Rioufol G, Finet G, Ginon I, Andre-Fouet X, Rossi R, Vialle E, Desjoyaux E, Convert G, Huret JF, Tabib A. Multiple atherosclerotic plaque rupture in acute coronary syndrome: a three-vessel intravascular ultrasound study. Circulation 2002;106:804-808.

Schaar JA, de Korte CL, Mastik F, van Damme LC, Krams R, Serruys PW, van der Steen AF. Three-dimensional palpography of human coronary arteries. Ex vivo validation and in-patient evaluation. Herz 2005;30:125-133.

Virmani R, Kolodgie FD, Burke AP, Farb A, Schwartz SM. Lessons from sudden coronary death: a comprehensive morphological classification scheme for atherosclerotic lesions. Arterioscler Thromb Vasc Biol 2000;20:1262-1275.

Virmani R, Burke AP, Farb A, Kolodgie FD. Pathology of the vulnerable plaque. J Am Coll Cardiol 2006;47:C13-C18.

Waters KR, Bautista R, Zelenka R, Masters D, Reynolds JS, Nelson S, Lam DH, Neville R, Moore TC. Development of a high-definition intravascular ultrasound imaging system and catheter. IEEE International Ultrasonics Symposium Proceedings 2011;1762-1765.

Williamson SD, Lam Y, Younis HF, Huang H, Patel S, Kaazempur-Mofrad MR, Kamm RD. On the sensitivity of wall stresses in diseased arteries to variable material properties. J Biomech Eng 2003;125:147-155.

Yla-Herttuala S, Bentzon JF, Daemen M, Falk E, Garcia-Garcia HM, Herrmann J, Hoefer I, Jukema JW, Krams R, Kwak BR, Marx N, Naruszewicz M, Newby A, Pasterkamp G, Serruys PW, Waltenberger J, Weber C, Tokgozoglu L. Stabilisation of atherosclerotic plaques. Position paper of the European Society of Cardiology (ESC) Working Group on atherosclerosis and vascular biology. Thromb Haemost 2011;106:1-19. 